\title{
Los bosques de Austrocedrus chilensis en Argentina: ubicación, estructura y crecimiento
}

\author{
Austrocedrus chilensis forest in Argentina: distribution, structure and stand growth
}

C.D.O.: 228

ALEJANDRO DEZZOTTI y LUIS SANCHOLUZ

Centro Regional Universitario Bariloche, Universidad Nacional

del Comahue, C.C. 1336 (8400), Bariloche, Argentina

\section{SUMMARY}

This study proposes a classification of Argentina's cypress forests into three types: marginal, compact and mixed forests (the latter with Nothofagus spp.). The classification is based on the information from seventy eight plots of cypress forest. Significative differences were found in density, basal areas and tree heights among forest types.

Analysis of the allometric relationship of the trees included in this study shows that: 1) the existing linear relationships between diameter and age are not that close, and 2) height growth is faster in the first years of development than in the later ones. Size distribution of the compact and mixed forest stands show inverted "J". Finally a positive linear relationship was found between stand height and mean annual volume increment. From the latter, it could be concluded that stand height could indicate site quality of cypress forests.

\section{RESUMEN}

En este trabajo se propone una clasificación de los bosques de ciprés de Argentina en tres tipos forestales: bosques marginales, bosques compactos y bosques mixtos (con Nothofagus spp.). Esta clasificación está basada en la información contenida en relevamientos de setenta y ocho parcelas de bosques de ciprés.

Se encontraron diferencias significativas entre los tipos forestales para sus densidades, áreas básales y alturas medias de los árboles. El análisis de las relaciones alométricas de los árboles comprendidos en este estudio indica que: 1) las relaciones lineales existentes entre diámetro y edad no son muy estrechas, y 2) el crecimiento en altura es más rápido en los primeros años de desarrollo que en los últimos. Las distribuciones de las tallas encontradas en los rodales analizados muestran "J" invertidas en los bosques compactos y mixtos. Por último, se encontró una relación positiva entre la altura del rodal y su crecimiento volumétrico medio anual. De esto se podría concluir que la altura del rodal sería un indicador de la calidad del sitio de los bosques de ciprés.

\section{INTRODUCCION}

Los Bosques Andino-Patagónicos en Argentina se caracterizan por un fuerte gradiente de precipitación ascendente Este-Oeste (Dimitri, 1972). En las zonas más húmedas del Oeste dominan las especies de Nothofagus; hacia el Este son reemplazadas por los bosques siempreverdes de ciprés de la cordillera (Austrocedrus chilensis (D. Don) Florin et Boutelje). La ecología y la biogeografía de estos bosques han sido discutidas por diversos autores (Dimitri, 1972; Eskuche, 1973; McQueen, 1976; Gallopín, 1978; Hueck, 1978, y Pearson y Pearson, 1982).

El ciprés de la cordillera es una especie endémica de la región Andino-Patagónica y la conífera de mayor rango geográfico de Argentina. Ha sido y es un importante recurso forestal para el desarrollo de la región, ya que los bosques de ciprés producen madera de buena calidad, leña, forraje para la ganadería cordillerana y microambientes favorables para los asentamientos humanos y las actividades turísticas. La información básica publicada sobre la especie misma se encuentra dispersa en trabajos de distinta índole: acerca de su distribución y silvicultura (Costantino, 1949,1958; Arschanov, 1967; Cozzo, 1979; Seibert, 1982; Mermoz y Martín, 1986), de su ecología (Lebedeff, 1942; Krebs, 1959; Thomasson, 1959; Havrylenko, 1965; Singer, 1971; Eskuche, 1973; Markgraf y D'Antoni, 1978; Lamarche et al., 1979; Rapoport y Drausal, 1979; Veblen y Lorenz, 1987, 1988), 
de su estado sanitario (Varsavsky et al., 1975; Havrylenko et al., 1990; Rosso et al., 1990; Fontenla et al., 1991), de su genética (Hunziker, 1961) y de sus usos alternativos (Fiaño, 1971; Rique et al., 1981). En este trabajo se recopila la información disponible sobre la distribución geográfica, estructura poblacional y crecimiento de los bosques naturales de ciprés en Argentina. Los objetivos de este análisis son: 1) clasificar los bosques espontáneos de ciprés en tipos forestales y ubicarlos geográficamente, 2) establecer los valores promedio de variables estructurales y de crecimiento del ciprés para cada tipo y analizar sus variaciones y, 3) discutir las relaciones entre las variables que caracterizan el estado y la dinámica de los bosques de ciprés.

\section{MATERIAL Y METODO}

La información sobre la distribución actual del ciprés en los Parques Nacionales (Lanín, Nahuel Huapi, Lago Puelo y Los Alerces) y Provincias Norpatagónicas de Argentina (Neuquén, Río Negro y Chubut) se obtuvo consultando los mapas de vegetación de Correa Luna (1969), Correa Luna y Dimitri (1969), Rodríguez García et al. (1978), Seibert (1982), López Cepero y Pintos (1984), APN (1980, 1986a, b), Mermoz y Martín (1987), Salguero (1990) y la precarta forestal (Chubut y Río Negro) del Instituto Forestal Nacional.

En todos estos trabajos se presentan clasificaciones particulares de los bosques de ciprés que son redefinidas para los fines de este trabajo. La clasificación que aquí se propone se basa en la ubicación geográfica de cada unidad de vegetación, en la precipitación media del área, en la diversidad de especies arbóreas y en las características generales de la unidad (cuadro 1). Los bosques mixtos de esta clasificación son asociaciones arbóreas entre A. chilensis y las especies de Nothofagus (principalmente $N$. dombeyi, pero también $N$. alpina y $N$. obliqua) siempre que, tanto la conífera como las latifoliadas, alcancen proporciones superiores al $20 \%$ del área basal total del rodal. Queda excluida de esta clasificación, por falta de información de base, la porción oriental de la provincia de Neuquén.

La información sobre la estructura y crecimiento de los bosques de ciprés se obtuvo de trabajos publicados (Lebedeff, 1942; Costantino, 1958; Graziosi, 1983; Veblen y Lorenz, 1987, 1988;
Dezzotti, 1989), de informes internos de la Universidad Nacional del Comahue y de trabajos de ordenación forestal. Todos estos trabajos contienen datos de setenta y ocho parcelas con bosques de ciprés relevadas con diferentes objetivos y métodos entre 1942 y 1991. Las parcelas se encuentran entre $\operatorname{los} 40^{\circ} 10^{\prime} \mathrm{y} 41^{\circ} 45^{\prime}$ lat. S. y entre los $71^{\circ}$ $06^{\prime}$ y $71^{\circ} 32^{\prime}$ long. O., con rangos de altitud entre 300 y $1.050 \mathrm{msnm}$ y de precipitación anual entre 700 y $1.800 \mathrm{~mm}$.

Para establecer los patrones de la estructura y el crecimiento del bosque en cada una de estas parcelas sólo fueron considerados los cipreses adultos (> $10 \mathrm{~cm}$ de diámetro a la altura del pecho, DAP) y se seleccionaron como variables discriminatorias la densidad de árboles (ind/ha), el área basal $\left(\mathrm{m}^{2} /\right.$ ha), el volumen de madera $\left(\mathrm{m}^{3} / \mathrm{ha}\right)$, la altura media de los árboles $(\mathrm{m})$, el crecimiento volumétrico de madera $\left(\mathrm{m}^{3} / \mathrm{ha} /\right.$ afio $)$ y el incremento diametral $(\mathrm{mm} /$ año). En el texto se citan medias \pm desviaciones estándar. La estructura de las tallas de los árboles se analizó a través de la distribución de las frecuencias de las clases de diámetro (clase 1:10$19.9 \mathrm{~cm}$ DAP, 2:20-29.9,.., 6:>60 cm DAP).

Mediante el análisis de la varianza se analizaron las variables de estudio en los diferentes tipos forestales, y con la Prueba de Comparaciones Múltiples de Scheffé para un diseño completamente aleatorizado (Hicks, 1973) se establecieron grupos homogéneos entre los tipos forestales; en ambos casos se tomaron $\mathrm{P}<0.05$. Utilizando técnicas de regresión simple, lineales y no lineales, se estudiaron las relaciones matemáticas entre el diámetro, la edad y la altura de los árboles en cada parcela. Utilizando la misma técnica se evaluaron las relaciones entre la densidad, la biomasa y el crecimiento del rodal de cada tipo forestal. En todos los casos se seleccionó el mejor modelo de todos los probados mediante el criterio del mayor coeficiente de determinación $\mathrm{R}^{2}$.

\section{RESULTADOS Y DISCUSION}

Los bosques de ciprés conforman un "cinturón de frontera" entre dos regiones ecológicas con características bien definidas: al Este, la estepa patagónica y, al Oeste, los bosques húmedos de Nothofagus. En respuesta a la alta heterogeneidad ambiental de esta región, las poblaciones naturales de ciprés se comportan en forma diferente, pudiéndose identificar de Este a Oeste los siguientes tipos 


\section{CUADRO 1}

Características de los tipos forestales de ciprés

Characteristics of the types of cypress forest

\begin{tabular}{|c|c|c|c|}
\hline $\begin{array}{l}\text { Tipo de } \\
\text { bosque }\end{array}$ & Características generales & $\begin{array}{l}\text { Comunidad } \\
\text { dominante }\end{array}$ & $\begin{array}{l}\text { Lluvia } \\
\text { (mm/año) }\end{array}$ \\
\hline $\begin{array}{l}\text { Marginal } \\
\quad(\mathrm{BM})\end{array}$ & $\begin{array}{l}\text { Individuos aislados o en pequeños } \\
\text { grupos, preferentemente en la cima } \\
\text { de lugares elevados. }\end{array}$ & $\begin{array}{l}\text { Estepa } \\
\text { graminosa } \\
\text { arbustiva }\end{array}$ & $600-900$ \\
\hline $\begin{array}{l}\text { Compacto } \\
\text { (BC) }\end{array}$ & $\begin{array}{l}\text { Bosques puros de ciprés ralos a den- } \\
\text { sos dependiendo de las condiciones } \\
\text { locales. }\end{array}$ & Bosque & $900-1.600$ \\
\hline $\begin{array}{l}\text { Mixto } \\
(\mathrm{BX})\end{array}$ & $\begin{array}{l}\text { Bosques mixtos de ciprés y Nothofa- } \\
\text { gus spp., la composición relativa de } \\
\text { ambas especies depende de las con- } \\
\text { diciones locales. }\end{array}$ & Bosque & $1.600-2.000$ \\
\hline
\end{tabular}

boscosos: a) bosques marginales (BM), b) bosques compactos (BC) y c) bosques mixtos (BX).

El cuadro 1 presenta las características de los tres tipos de bosques de ciprés identificados en este estudio. Entre las isoyetas de 600 y $900 \mathrm{~mm} /$ año se desarrollan los BM, constituidos por cipreses aislados o reunidos en pequeños grupos, de baja densidad, biomasa y crecimiento. Se ubican, generalmente, en las colinas rocosas, donde la rugosidad del terreno ofrecería micrositios reparados al desecamiento y donde la presión del pastoreo ovino sería menor que en las áreas esteparias circundantes. Entre las isoyetas de 900 y 1.600 $\mathrm{mm} / \mathrm{año}$ se encuentran los BC, monoespecíficos, donde el ciprés forma rodales más o menos cerrados dependiendo de las condiciones locales. En las laderas más secas, en pendientes fuertes o en suelos rocosos el ciprés forma un bosque abierto con densidad, cobertura y productividad menor a la observada en las laderas más húmedas. Entre los 1.600 y $2.000 \mathrm{~mm}$ de precipitación anual el ciprés forma los $\mathrm{BX}$ y entra en fuerte competencia con las especies de Nothofagus que siempre dominan el dosel. La proporción de ciprés, en la composición del rodal, varía en forma inversa a las condiciones de humedad del sitio.

En el mapa de la figura 1 se presenta la distribución geográfica de ciprés en Argentina. Estos bosques se extienden en una franja discontinua desde $\operatorname{los} 36^{\circ} 30^{\prime}$ lat. S. (río Neuquén Norte, Neuquén) (Seibert 1982) y hasta $\operatorname{los} 43^{\circ} 35^{\prime}$ lat. S. (cuenca del río Corcovado, Chubut). El límite altitudinal superior de esta especie se encuentra a los 1.000-1.100 msnm y los factores restrictivos serían su sensibilidad a las bajas temperaturas y el daño que la nieve causa en sus renuevos (Eskuche, 1973). Aunque en Chile los bosques de ciprés presentan una extensión latitudinal y altitudinal mayor, extendiéndose desde los $32^{\circ} 39^{\prime}$ hasta los $44^{\circ}$ lat. Sur (Schlegel, 1962; Hueck, 1978) y hasta los $1.800 \mathrm{msnm}$ (Serra et al., 1986), las superficies cubiertas son menores (Donoso, 1983).

Los bosques de ciprés mapeados ocupan alrededor de 159.000 ha, distribuidas en las diferentes jurisdicciones es de acuerdo al cuadro 2. Los BM y los BX cubren 31.000 ha cada uno (19.5\% cada uno del total) y los BC 97.000 ha $(61 \%)$. Alrededor de 89.500 ha de ciprés se encuentran protegidos por la Administración de Parques Nacionales (56.3\% del total), siendo la categoría más protegida la de los BC (47.000 ha, $48.5 \%$ del tipo forestal), luego siguen los BM (24.700 ha, 79.7\%) y finalmente los BX (17.800 ha, 57.4\%). La provincia de Chubut cuenta con la mayor superficie con ciprés (45.300 ha) y le sigue el Parque Nacional Nahuel Huapi con 36.200 ha. En relación a su superficie, los Parques Nacionales Lago Puelo y Lanín son las jurisdicciones con mayor cantidad de cipresales ( $19.5 \%$ y $7.3 \%$, respectivamente).

En el cuadro 3 se detallan las características estructurales de los distintos tipos forestales de ciprés, según surge del análisis de las parcelas de estudio. En los BM la densidad media de árboles es $268 \pm 136 \mathrm{ind} / \mathrm{ha}$, el área basal es $12.1 \pm 10.7$ $\mathrm{m}^{2} /$ ha y el volumen $57.3 \pm 54.9 \mathrm{~m}^{3} / \mathrm{ha}$. En los $\mathrm{BC}$ la densidad es, en promedio, $798 \pm 427 \mathrm{ind} / \mathrm{ha}$, el 


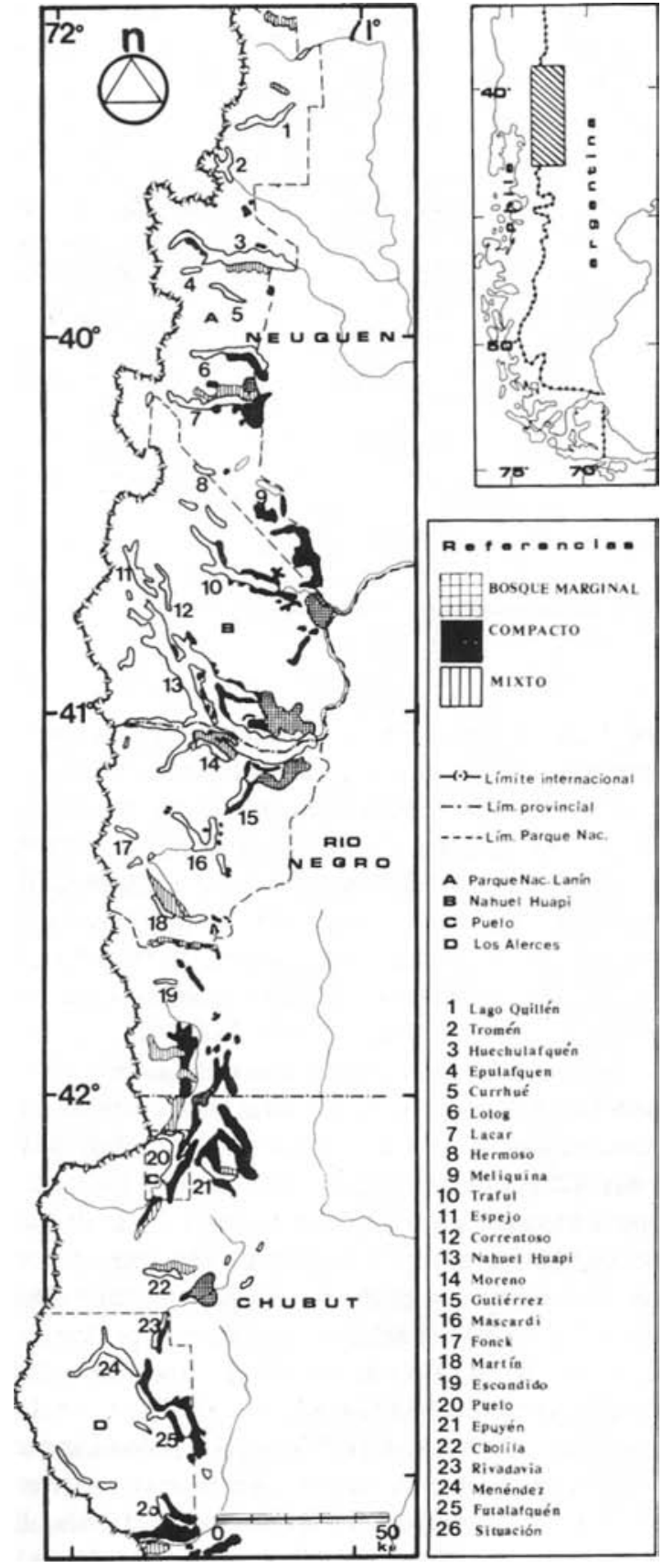

Fig. 1. Mapa de distribución de los bosques de ciprés en la Argentina, indicando la ubicación de los tres tipos forestales propuestos en este estudio.

Distribution of the three Argentinian cypress forest types proposed.

área basal $55.6 \pm 25.4 \mathrm{~m}^{2} / \mathrm{ha}$ y el volumen $286.9 \pm$ $\mathrm{m}^{3} / \mathrm{ha}$. En los BX en promedio se encuentran 460 \pm 316 individuos en ciprés $/ \mathrm{ha}, 36.7 \pm 22.3 \mathrm{~m}^{2} / \mathrm{ha} \mathrm{de}$ área basal y $330.6 \pm 173.5 \mathrm{~m}^{3}$ de madera/ha. El crecimiento volumétrico promedio de los BM es
1.2, en los BC 3.7 y en los BX $3.3 \mathrm{~m}^{3} / \mathrm{ha} / \mathrm{año}$. El incremento diametral promedio es $5.6,3.8$ y 3.3 $\mathrm{mm} / \mathrm{año}$ en los BX, BC у BM, respectivamente. La mayor altura media de los cipreses se observa en los BX con $22.9 \pm 4.9 \mathrm{~m}$, valor que decrece hacia el Este, con $8.1 \pm 2.9 \mathrm{~m}$ en los $\mathrm{BM}$.

Los bosques estudiados presentan diferencias significativas entre los promedios de la densidad, el área basal y la altura del rodal. Los BC registran los valores más altos de densidad y área basal, mientras que los más bajos se observan en los BM. El volumen, en cambio, no difiere significativamente en $\operatorname{los} \mathrm{BC}$ y BX, debido a que la mayor altura media de los árboles de este último tipo compensaría su menor área basal. Con respecto a los ritmos de crecimiento, los BM presentan los menores valores de crecimiento volumétrico y los mayores de incremento diametral; estos valores difieren significativamente de los registrados en los BC y BX, que forman entre sí un grupo homogéneo para ambas variables.

Pese a que el ciprés es un árbol longevo (Tortorelli, 1956; La Marche et al., 1979), la edad máxima media de los individuos en las parcelas de los BC estudiados (de las otras categorías no se contó con suficiente información) es $96 \pm 43$ años, con un rango que varía entre 27 y 189 años ( $=30$ árboles). La distribución que sigue esta variable es normal (test de bondad de ajuste KolmogorovSmirnov, $\mathrm{P}<0.05)$. La edad media máxima de los BC de ciprés coincide con la colonización europea de la región, que, entre otras cosas, produjo incendios forestales a gran escala.

El cuadro 4 presenta los resultados de los estudios alométricos realizados en este trabajo. El modelo matemático que mejor ajusta las relaciones entre la edad y el diámetro de los árboles en todas las parcelas con datos (17) es lineal $(\mathrm{Y}=\mathrm{a}+\mathrm{bX})$. Una parcela mostró el mejor ajuste con un modelo multiplicativo $\left(Y=a X^{b}\right)$ y en cinco estaciones las relaciones no fueron estadísticamente significativas. Los coeficientes de determinación $\mathrm{R}^{2}$ varían entre 0.28 y 0.94 , indicando pobres a buenos ajustes. En general, los modelos lineales y multiplicativos son los que mejor ajustan la dependencia entre la edad y la altura de los árboles, variando al $\mathrm{R}^{2}$ entre 0.58 y 0.97 , y con una relación no significativa en una estación. Aunque en general las relaciones entre el número de anillos de crecimiento y el diámetro y la altura fueron significativas, cada clase de tamaño contiene un rango amplio de diferentes edades. La independencia o las pobres rela- 
CUADRO 2

Superficies cubiertas por los tipos forestales de ciprés (en ha) en las Provincias y Parques Nacionales de Argentina

Areas covered with types of cypress forest (ha) in the Provinces and National Parks of Argentina

\begin{tabular}{|lcccrrr|}
\hline & \multicolumn{3}{c}{ TIPO DE BOSQUE } & & \\
\cline { 2 - 4 } Jurisdicción & $\begin{array}{c}\text { Marginal } \\
\text { (BM) }\end{array}$ & $\begin{array}{c}\text { Compacto } \\
(\text { BC) }\end{array}$ & $\begin{array}{c}\text { Mixto } \\
(\mathrm{BX})\end{array}$ & TOTAL & $\%^{2}$ \\
\hline Provincia Río Negro & & 20.000 & 4.200 & 24.200 & 0.1 \\
Provincia Chubut & 6.300 & 30.000 & 9.000 & 45.300 & 0.2 \\
Parque Nacional Lanín & 14.500 & 7.000 & 6.200 & 27.700 & 7.3 \\
Parque Nac. Nahuel Huapi & 10.200 & 15.000 & 11.000 & 36.200 & 4.6 \\
Parque Nac. Lago Puelo & - & 4.000 & 600 & 4.600 & 19.5 \\
Parque Nac. Los Alerces & - & 21.000 & - & 21.000 & 8.0 \\
TOTAL & 31.000 & 97.000 & 31.000 & 159.000 & 0.3 \\
\hline
\end{tabular}

${ }^{1}$ No incluye los bosques de ciprés de los Parques Nacionales.

${ }^{2}$ Porcentaje con ciprés en relación a la superficie de la jurisdicción.

\section{CUADRO 3}

Identificación de grupos homogéneos (GH) entre los tipos de bosques de ciprés, utilizando el test de rango múltiple de Scheffé $(\mathrm{P}<0.05)$. Se indican los valores medios (X), el número de muestras (n) y la desviación estándar (DE) de la densidad del área basal, del volumen, de la altura, del crecimiento volumétrico y diametral. Letras iguales (a, b y c) indican grupos homogéneos

Identification of homogeneous groups (GH) among types of cypress forests using Scheffé's multiple range test $(\mathrm{P}<0.05)$. Means $(X)$, sample size (n) and standard deviations (DE) are shown for density, basal area, volume height, volume and diameter increment. Same letters ( $\mathrm{a}, \mathrm{b}$ and $\mathrm{c}$ ) indicate homogeneous groups

\begin{tabular}{|clrrr|}
\hline Variable & & $\begin{array}{c}\text { Bosque } \\
\text { Marginal } \\
(\mathrm{BM})\end{array}$ & $\begin{array}{c}\text { Bosque } \\
\text { Compacto } \\
(\mathrm{BC})\end{array}$ & $\begin{array}{r}\text { Bosque } \\
\text { Mixto } \\
(\mathrm{BX})\end{array}$ \\
\hline & $\mathrm{X}$ & 268 & 798 & 460 \\
Densidad & $\mathrm{DE}$ & 136 & 428 & 316 \\
(ind/ha) & $\mathrm{GH}$ & 6 & 53 & 18 \\
& $\mathrm{X}$ & 12.1 & 55.6 & 36.7 \\
& $\mathrm{DE}$ & 10.7 & 25.4 & 22.3 \\
Area basal & $\mathrm{n}$ & 6 & 51 & 18 \\
(m²/ha) & $\mathrm{GH}$ & $\mathrm{a}$ & $\mathrm{b}$ & $\mathrm{c}$ \\
& $\mathrm{X}$ & 57.3 & 286.9 & 330.6 \\
& $\mathrm{DE}$ & 54.8 & 197.6 & 173.5 \\
Volumen & $\mathrm{n}$ & 6 & 23 & 3 \\
(m ${ }^{3} /$ ha) & $\mathrm{GH}$ & $\mathrm{a}$ & $\mathrm{b}$ & $\mathrm{b}$ \\
& $\mathrm{X}$ & 8.1 & 13.3 & 22.9 \\
& $\mathrm{DE}$ & 2.9 & 3.9 & 4.9 \\
Altura & $\mathrm{n}$ & 6 & 16 & 3 \\
(m) & $\mathrm{GH}$ & $\mathrm{a}$ & $\mathrm{b}$ & $\mathrm{c}$ \\
& $\mathrm{X}$ & 1.2 & 3.7 & 3.3 \\
Crecimiento & $\mathrm{DE}$ & 1.1 & 2.1 & 1.4 \\
Volumétrico & $\mathrm{n}$ & 6 & 14 & 3 \\
(m ${ }^{3} /$ há/año) & $\mathrm{GH}$ & $\mathrm{a}$ & $\mathrm{b}$ & $\mathrm{b}$ \\
& $\mathrm{X}$ & 5.6 & 3.8 & 3.3 \\
Crecimiento & $\mathrm{DE}$ & 2.2 & 1.1 & 1.3 \\
diametral & $\mathrm{n}$ & 6 & 16 & 3 \\
(mm/año) & $\mathrm{GH}$ & $\mathrm{a}$ & $\mathrm{b}$ & $\mathrm{b}$ \\
\hline & & & & \\
\hline
\end{tabular}

ciones observadas entre estas variables reflejaría, por un lado, la capacidad de esta especie de persistir aun como árbol suprimido (Veblen y Lorenz, 1987), y por el otro, que los cipreses más viejos desarrollaron tasas de crecimiento mayores al establecerse en condiciones de baja competencia. Los modelos multiplicativos (15 parcelas) y los lineales ( 8 parcelas) muestran un buen ajuste de las variables diámetro y altura, con $\mathrm{R}^{2}$, variando entre 0.17 y 0.98 , y con todas las relaciones significativas.

En general se observa que la tasa de acumulación de área basal a lo largo del ciclo de vida del ciprés permanece constante para un sitio en particular. Esto se evidencia en la relación lineal que prevalece entre la edad y el diámetro. Las pendientes (b) de las funciones para las estaciones estudiadas difieren significativamente entre sí $(\mathrm{P}<0.05)$ (b varía entre 0.18 y 2.21$)$, lo que indicaría que existe un efecto del sitio sobre la velocidad de crecimiento del diámetro de los árboles. Con relación al crecimiento en altura se observa que en general es mayor en las etapas juveniles del desarrollo de los cipreses, puesto que domina el modelo multiplicativo entre la edad y la altura de los árboles. Las pendientes de las funciones entre estas variables difieren significativamente entre estaciones (b varía entre 0.02 y 1.76), indicando también que hay un efecto del sitio sobre la altura. Como era de esperar de las relaciones lineales observadas entre la edad y el diámetro, se ve que generalmente los árboles de menores tallas crecen más rápidamente en altura; las pendientes de las funciones ajustadas varían entre 0.02 y 0.81 y difieren significativamente entre sí $(\mathrm{P}<0.05)$.

También se evaluó la relación existente entre la altura de los árboles para cada estación, estimada 
a partir de las ecuaciones ajustadas para el diámetro y la altura, utilizando un DAP igual a $20 \mathrm{~cm}$, y el crecimiento volumétrico para las ocho parcelas de los BC que cuentan con datos para ambas variables. Si bien el número de muestras es pequeño, se observa una correlación positiva alta $(\mathrm{R}=0.84)$ entre estas variables. La altura de los árboles de ciprés podría ser entonces un indicador del crecimiento y de la capacidad productiva del sitio forestal, éste definido por la combinación de variables físicas, edáficas y climáticas. Del mismo modo, analizando conjuntamente las mismas variables presentadas en el cuadro 4, altura media y crecimiento volumétrico de los tres tipos de bosques de ciprés, se observa que existe una relación entre ambas. Considerando como unidad el sitio forestal, los BM presentan los menores valores de estas variables, mientras que los $\mathrm{BX}$ presentarían los mayores valores si se evalúa no sólo el aporte del ciprés a la productividad sino también el del coihue ( $N$. dombeyi), que no fue determinado en este estudio.

El cuadro 5 presenta los análisis de regresión simple $(\mathrm{P}<0.05)$ entre las variables densidad, área basal, volumen, crecimiento volumétrico e incremento diametral, tomadas como el total para cada parcela. Ajustadas a través de un modelo exponencial $\left(y=e^{(a+b x)}\right.$, se observan relaciones fuertes

\section{CUADRO 4}

Coeficientes de determinación (R2) para los modelos de regresión simple ( $\mathrm{P}<0.05)$, entre las variables diámetro (DAP), edad y altura (Alt), para cada rodal con ciprés

Determination coefficient (R2) for the single regression models $(\mathrm{P}<0.05)$ among diameter (DAP), age (edad) and height (Alt) for every cypress stand.

\begin{tabular}{|c|c|c|c|c|c|c|c|}
\hline Parcela & $\begin{array}{c}\text { Edad/DAP } \\
\text { (R2) }\end{array}$ & $\mathrm{n}$ & $\begin{array}{l}\text { DAP/alt } \\
\text { (R2) }\end{array}$ & $\mathrm{n}$ & $\begin{array}{l}\text { Edad/alt } \\
\text { (R2) }\end{array}$ & $\mathrm{n}$ & $\begin{array}{c}\text { Bosque } \\
\text { Tipo }\end{array}$ \\
\hline Cerro Leones & 0.42 & 24 & 0.24 & 80 & & & $\mathrm{BM}$ \\
\hline Confluencia & 0.91 & 24 & 0.60 & 79 & & & $\mathrm{BC}$ \\
\hline Cerro Otto & 0.28 & 25 & 0.60 & 80 & & & $\mathrm{BC}$ \\
\hline Cerro Runge & 0.55 & 25 & 0.60 & 80 & & & $\mathrm{BC}$ \\
\hline Isla Gallina & $\mathrm{ns}$ & 10 & 0.17 & 78 & & & $\mathrm{BC}$ \\
\hline Gutiérrez 1 & 0.77 & 25 & 0.80 & 80 & & & $\mathrm{BC}$ \\
\hline Mascardi A & 0.45 & 20 & 0.46 & 77 & & & $\mathrm{BX}$ \\
\hline Loma Medio & $\mathrm{ns}$ & 13 & 0.90 & 13 & $\mathrm{~ns}$ & 13 & $\mathrm{BC}$ \\
\hline Gutiérrez 3 & $\mathrm{~ns}$ & 6 & 0.91 & 6 & 0.72 & 6 & $\mathrm{BC}$ \\
\hline Neuquén la & $\mathrm{ns}$ & 14 & & & & & $\mathrm{BM}$ \\
\hline Neuquén 2a & 0.91 & 40 & & & & & $\mathrm{BM}$ \\
\hline Neuquén 3a & 0.86 & 40 & & & & & $\mathrm{BM}$ \\
\hline Neuquén $4 \mathrm{a}$ & 0.66 & 46 & & & & & $\mathrm{BM}$ \\
\hline Neuquén 5a & 0.71 & 40 & & & & & $\mathrm{BM}$ \\
\hline Neuquén $6 \mathrm{a}$ & 0.61 & 44 & & & & & $\mathrm{BC}$ \\
\hline Neuquén $7 \mathrm{a}$ & 0.44 & 42 & & & & & $\mathrm{BC}$ \\
\hline Neuquén 9a & 0.87 & 39 & & & & & $\mathrm{BC}$ \\
\hline Neuquén 8 a & 0.40 & 45 & & & & & $\mathrm{BC}$ \\
\hline S.M. Andes 1 & 0.95 & 7 & 0.99 & 7 & 0.96 & 7 & $\mathrm{BC}$ \\
\hline S.M. Andes 2 & 0.94 & 6 & 0.95 & 6 & 0.99 & 6 & $\mathrm{BC}$ \\
\hline S.M. Andes 3 & 0.93 & 7 & 0.98 & 7 & 0.97 & 7 & $\mathrm{BC}$ \\
\hline Bariloche 1 & & & 0.32 & 31 & & & $\mathrm{BC}$ \\
\hline Bariloche 2 & & & 0.38 & 182 & & & $\mathrm{BC}$ \\
\hline Llao Llao A & 0.68 & 15 & 0.60 & 45 & 0.58 & 15 & $\mathrm{BC}$ \\
\hline Llao Llao B & 0.68 & 15 & 0.68 & 67 & 0.58 & 15 & $\mathrm{BC}$ \\
\hline Llao Llao C & 0.68 & 15 & 0.59 & 28 & 0.58 & 15 & $\mathrm{BC}$ \\
\hline Limay A & $\mathrm{ns}$ & 11 & 0.81 & 18 & 0.59 & 11 & $\mathrm{BC}$ \\
\hline Limay B & $\mathrm{ns}$ & 11 & 0.86 & 31 & 0.59 & 11 & $\mathrm{BC}$ \\
\hline Limay C & $\mathrm{ns}$ & 11 & 0.83 & 37 & 0.59 & 11 & $\mathrm{BC}$ \\
\hline Epuyén A & & & 0.95 & 13 & & & $\mathrm{BC}$ \\
\hline Epuyén B & & & 0.92 & 13 & & & $\mathrm{BC}$ \\
\hline Epuyén $\mathrm{C}$ & & & 0.98 & 13 & & & $\mathrm{BC}$ \\
\hline Epuyén $T$ & & & 0.96 & 6 & & & $\mathrm{BC}$ \\
\hline
\end{tabular}




\section{CUADRO 5}

Análisis de regresión simple entre las variables Area basal (AB), Volumen (VOL),

densidad (DEN), crecimiento volumétrico (CV) y diametral (CD) para los tres tipos de bosques (TB).

Se indica el modelo que mejor ajusta $(\mathrm{P}<0.05)$, el valor de los parámetros A (intercepto), B (pendiente), el coeficiente de determinación (R2) y el número de muestras (n)

Single regression analysis for variables basal area (AB), volume (VOL), density (DEN), volumetric growth (CV) and diameter (CD). Increment for the three forest types (TB). The best model $(\mathrm{P}<0.05)$ with its parameters A (intercept) and B (slope), and the sample size (n) is show

\begin{tabular}{|c|c|c|c|c|c|c|c|}
\hline $\mathrm{X}$ & $\mathrm{Y}$ & Modelo & A & B & $\mathrm{R} 2$ & $\mathrm{~N}$ & TB \\
\hline $\mathrm{AB}$ & VOL & Exponencial & 2.38 & 1.25 & 0.99 & 6 & $\mathrm{BM}$ \\
\hline DEN & $\mathrm{CV}$ & Lineal & -0.83 & 0.01 & 0.89 & 6 & $\mathrm{BM}$ \\
\hline $\mathrm{AB}$ & VOL & Exponencial & 2.71 & 1.22 & 0.85 & 21 & $\mathrm{BC}$ \\
\hline VOL & $\mathrm{CV}$ & Lineal & 1.34 & 0.01 & 0.65 & 14 & $\mathrm{BC}$ \\
\hline VOL & $\mathrm{CD}$ & Exponencial & 45.87 & -0.47 & 0.57 & 15 & $\mathrm{BC}$ \\
\hline $\mathrm{AB}$ & $\mathrm{CV}$ & Exponencial & 0.14 & 0.85 & 0.62 & 13 & $\mathrm{BC}$ \\
\hline $\mathrm{AB}$ & $\mathrm{CD}$ & Exponencial & 17.12 & -0.42 & 0.42 & 15 & $\mathrm{BC}$ \\
\hline DEN & $\mathrm{CD}$ & Exponencial & 56.69 & -0.42 & 0.40 & 15 & $\mathrm{BC}$ \\
\hline DEN & $\mathrm{CV}$ & Lineal & 1.09 & 0.01 & 0.71 & 13 & $\mathrm{BC}$ \\
\hline DEN & $\mathrm{AB}$ & Lineal & 14.41 & 0.05 & 0.47 & 18 & $\mathrm{BX}$ \\
\hline
\end{tabular}

Nota: Modelo exponencial $\mathrm{Y}=\mathrm{e}^{(\mathrm{a}+\mathrm{bx})}$

Modelo lineal $\mathrm{Y}=\mathrm{a}+\mathrm{bx}$

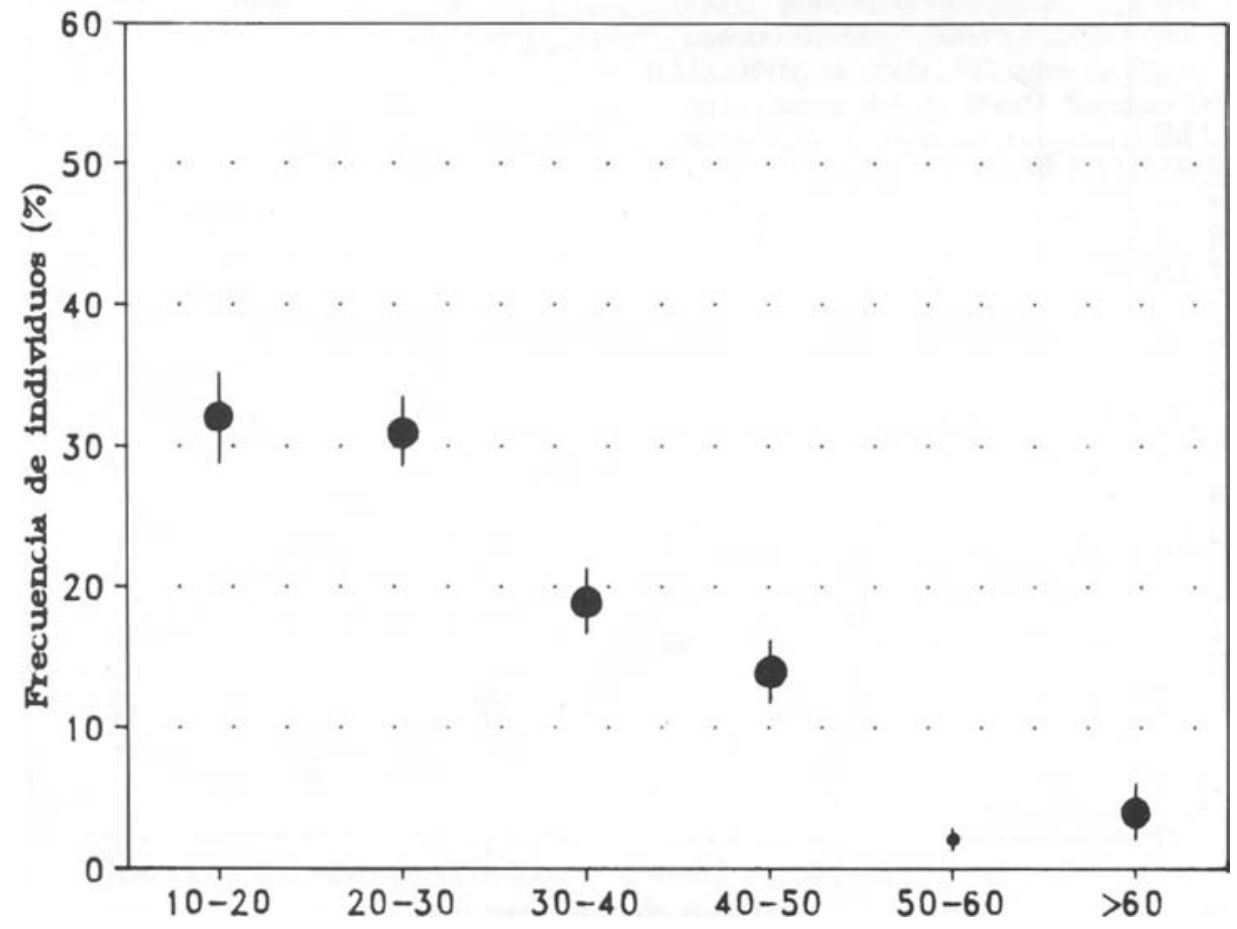

Fig. 2. Distribución de las frecuencias medias (\%) de las clases diamétricas para los Bosques Compactos de ciprés. La barra indica el error estándar de la media.

Average size class distribution (\%) of the cypress Compact Forests. Bar indicates mean standard error. 
entre el área basal y el volumen para los BM y los $\mathrm{BC}\left(\mathrm{R}^{2}=0.99\right.$ y 0.85 respectivamente); esto parece razonable ya que existe una dependencia entre el DAP y la altura. De este modo es posible utilizar el área basal como indicador del volumen, que es a veces difícil de medir. Para los BM no se contó con suficiente información sobre el comportamiento de estas variables. El crecimiento volumétrico muestra una dependencia con la densidad en ambos tipos forestales $\left(B M R^{2}=0.89\right.$ y $B C R^{2}$ $=0.71)$, como también con el volumen $\left(R^{2}=0.65\right)$ y el área basal $\left(\mathrm{R}^{2}=0.62\right)$ en $\operatorname{los} \mathrm{BC}$. Para una evaluación rápida del rendimiento del ciprés en una parcela de estudio podrían utilizarse estas dos últimas variables como indicadores. En los BC el incremento diametral varía inversamente con la densidad, el área basal y el volumen $\left(\mathrm{R}^{2}=0.40,0.42\right.$ y 0.57 , respectivamente). Aunque en un rodal el crecimiento volumétrico aumenta a medida que aumenta la densidad, el área basal y el volumen, los árboles analizados individualmente acumulan menor biomasa a medida que se incrementa la competencia por recursos. Sólo para los BX se encontraron relaciones pobres entre la densidad y el área basal $\left(\mathrm{R}^{2}=0.47\right)$.

La distribución de las frecuencias diamétricas se estudió para los BC y los BX, donde se contó con suficiente información (48 y 15 parcelas respectivamente). Se ajustaron modelos no lineales $(\mathrm{P}<0.05)$ entre las clases diamétricas xi $(\mathrm{i}=1$, $2, \ldots, 6)$ y la frecuencia media de individuos en porcentaje para cada clase, para ambas categorías de bosque. Se obtuvo una estructura de tallas de tipo exponencial negativa ("J" invertida), como puede verse en las figuras 2 y 3 . En ambos tipos forestales el $63 \%$ de los individuos totales del rodal se concentran en las clases 1 y 2 , y alrededor del $20 \%$ en las clases 3 y 4 . Aunque las frecuencias medias conforman "J" invertidas, existe variación alrededor de las medias de cada clase de tamaño.

\section{CONCLUSIONES}

En este estudio se caracterizaron y ubicaron geográficamente tres tipos de bosques de ciprés en

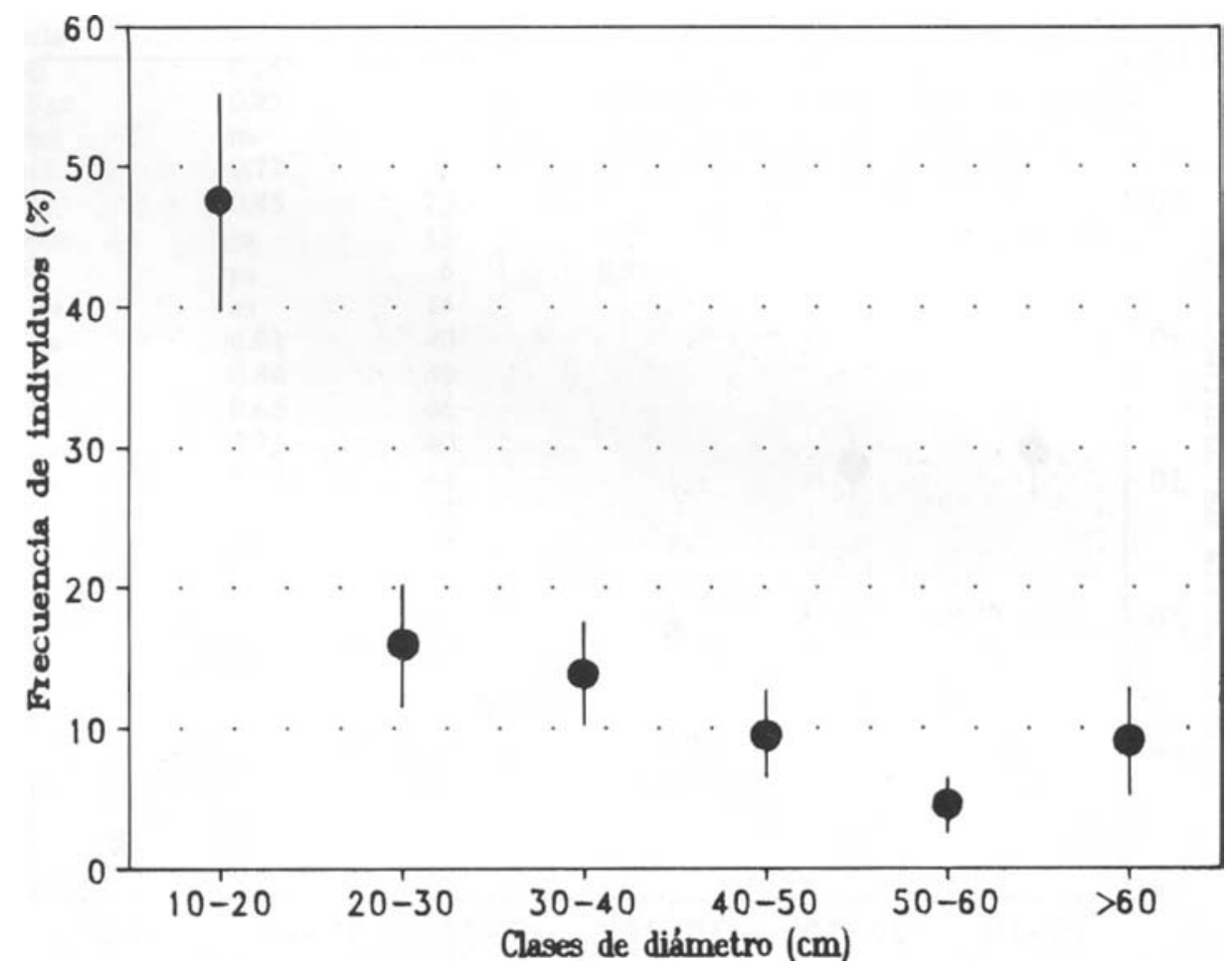

Fig. 3. Distribución de las frecuencias medias (\%) de las clases diamétricas para los Bosques Mixtos de ciprés. La barra indica el error estándar de la media.

Average size class distribution (\%) of the cypress Mixed Forests. Bar indicates mean standard error. 
Argentina. Estos ocupan 160.000 ha y presentan diferencias entre sí en cuanto a la densidad, el área basal media y la altura media de los árboles. Con respecto a las otras variables analizadas se observa que los bosques marginales presentan valores de volumen medio, crecimiento volumétrico e incremento diametral medio que difieren de los registrados en los bosques compactos y mixtos.

De los estudios alométricos presentados surge que sólo en algunos casos existen relaciones lineales significativas entre la edad y el diámetro de los árboles. La falta de un buen ajuste estadístico entre estas variables podría explicarse por la tolerancia del ciprés a la competencia que provocaría un retardo en su crecimiento. La distribución de las frecuencias de las clases de tamaño no puede ser usada entonces para inferir la estructura de edades del ciprés, que no pudo ser evaluada adecuadamente en este estudio.

Un subconjunto de las parcelas de estudio muestra que existe una relación lineal positiva entre la altura media de los árboles y el crecimiento volumétrico. La altura podría ser entonces un indicador de la productividad maderera de los bosques de ciprés, aun cuando convendría realizar observaciones más precisas.

\section{BIBLIOGRAFIA}

APN. 1980. Mapa de vegetación del Parque Nacional Lanín. Grupo de Relevamiento de Unidades Ecológicas. Administración de Parques Nacionales. Buenos Aires.

1986a. Plan general de manejo Parque Nacional Los Alerces. Documento de Avance. Administración de Parques Nacionales/Consejo Federal de Inversiones. Buenos Aires.

1986b. Plan general de maneja Parque Nacional Nahuel Huapi. Administración de Parques Nacionales/Consejo Federal de Inversiones. Buenos Aires.

ARSCHANOV, B. 1967. "Parcelas experimentales de Austrocedrus chilensis y Nothofagus dombeyi en la isla Victoria del Parque Nacional Nahuel Huapi, Argentina", Revista Forestal Argentina 11(2): 37-45.

CORREA LUNA, H. 1969. Cuenca del río Manso Superior. Parque Nacional Nahuel Huapi. Dirección General de Parques Nacionales/Fundación Bariloche. Buenos Aires.

CORREA LUNA, H. y DIMITRI, M. 1969. Las comunidades florísticas de la cuenca del río Manso Superior. Dirección General de Parques Nacionales/Fundación Bariloche. Buenos Aires.

COSTANTINO, I. 1949. Parcelas experimentales permanentes de Libocedrus chilensis (Don) Endl. Publicación Administración Nacional de Bosques $\mathrm{N}^{s}$ 13. Buenos Aires.
1958. "Primeros resultados de las parcelas permanentes de Libocedrus chilensis", Revista Facultad de Agronomía La Plata 34(2): 131-159.

COZZO, D. 1979. "Arboles forestales, maderas y silvicultura de la Argentina", Enciclopedia Argentina de Agricultura y Jardinería 2(16-1): 1-256.

DEZZOTTI, A. 1989. Distribución, estructura y dinámica de Austrocedrus chilensis bajo distintas condiciones ecológicas. Tesis de grado, Universidad Nacional del Comahue, Centro Regional Universitario Bariloche, $74 \mathrm{pp}$.

DIMITRI, M. 1972. La región de los bosques AndinoPatagónicos. Sinopsis General. Colección Científica Instituto Nacional de Tecnología Agropecuaria. Tomo X. Buenos Aires.

DONOSO, C. 1983. "Modificaciones del paisaje chileno a lo largo de la historia", Actas del Simposio Desarrollo y Perspectivas de las Disciplinas Forestales. Universidad Austral de Chile, Valdivia 1: 365 438.

ERIKSEN, W. 1970. "Kolonisation und tourismus in Ostpatagonien", Bonnes Geographische Abhandlunger 43: 290.

ESKUCHE, U. 1973. "Estudios fitosociológicos en el norte de Patagonia", Phytocoenología 1(1): 64-113.

FIAÑO, E. 1971. Preparaciones de pastas celulósicas al sulfato y a la soda, a partir del ciprés (Austrocedrus chilensis) y coihue (Nothofagus dombeyi). CICELPA, Buenos Aires.

FONTENLA, S., HAVRYLENKO, M. y ROSSO, P. 1991. "Micorrizas vesículo-arbusculares en Austrocedrus chilensis". Suelo y Planta 1: 251-255.

GALLOPLN, G. 1978. "Estudio ecológico integrado de la cuenca del río Manso Superior (Río Negro, Argentina). 1. Descripción general de la cuenca", Anales Parques Nacionales 14: 161-230.

GRAZIOSI, P. 1983. Comparación del crecimiento diamétrico en dos rodales de ciprés de la cordillera (Austrocedrus chilensis) en dos ambientes diferentes. Tesis de grado. Universidad Nacional del Comahue, Asentamiento Universitario San Martín de Los Andes, $40 \mathrm{pp}$.

HAVRYLENKO, D. 1965. "Contribución dendrológica al conocimiento del ciprés de la cordillera (Austrocedrus chilensis). Revista Forestal Argentina 9(3): 78.

HAVRYLENKO, M., ROSSO, P. y FONTENLA, S. 1990. "Caracterización de la mortalidad de Austrocedrus chilensis en Argentina", Bosque 10(1): 29-36.

HICKS, C. 1973. Fundamental concepts in the design of experiments. Holt, Rinehart and Winston, New York.

HUECK, K. 1978. Los bosques de Sudamérica. Sociedad Alemana de Cooperación Técnica GTZ, Eschborn.

HUNZIKER, J. 1961. "Estudios cromosómicos en $\mathrm{Cu}$ pressus y Libocedrus (Cupressaceae)", Revista de Investigaciones Agrícolas 15(2): 169-181.

KREBS, C. 1959. "Observaciones fenológicas sobre plantas indígenas del Parque Nacional Nahuel Huapi", Anales Parques Nacionales 8: 127-133. 
LAMARCHE, V., HOLMES, R., DONWIDDIE, P. y DREW, L. 1979. Tree-ring chronologies of the southern hemisphere, Laboratory of Tree-Ring Research, University of Arizona.

LEBEDEFF, N. 1942. "Rejuvenecimiento del ciprés e influencia de la ganadería". Boletín Forestal 193840. Dirección General de Parques Nacionales, Buenos Aires.

LOPEZ CEPERO, E. y PINTOS, S. 1984. Vegetación leñosa de las cuencas de los lagos Lacar y Lolog. Dirección General de Bosques y Parques Provinciales de Neuquén, Neuquén.

MARKGRAF, V. y D'ANTONI H. 1978. Pollen flora of Argentina. University of Arizona Press.

MC QUEEN, D. 1976. "The ecology of Nothofagus and associated vegetation in South America", Tuatara 22: 38-68.

MERMOZ, M. y MARTIN, C. 1987. Mapa de vegetación del Parque y la Reserva Nacional Nahuel Huapi. Administración de Parques Nacionales/Secretaría de Ciencia y Técnica, Buenos Aires.

PEARSON, O y PEARSON, A. 1982. "Ecology and biogeography of the southern rainforest of Argentina", Special Publication Pymatuning Laboratory of Ecology 6: 129-142.

RAPOPORT, E. y DRAUSAL, B. 1979. "Tácticos y estrategas $\mathrm{r}, \mathrm{K}$ y $\mathrm{SOS} "$. Tópicos de ecología contemporánea. En: RABINOVICH y G. HALFFTER, Cap. 6: 111-128. Fondo Cultura Económica. México.

RIQUE, T., DE SUCATTE, S., REPELli, R. y BLANCHET, J. 1981. "Recursos forestales de la precordillera patagónica: potencial fuente de materia prima para la industria celulósica-papelera", ClCELPA. Publicación técnica $\mathrm{N}^{0} 41$, Buenos Aires.

RODRIGUEZ GARCIA, D., SOURROUILlE, A., GALLOPIN, G. y MONTAÑA, C. 1978. "Estudio ecológico integrado de la cuenca del río Manso Superior. II. Tipos de Vegetación", Anales de Parques Nacionales 14: 231-240.

ROSSO, P., HAVRYLENKO, M. y FONTENLA, S. 1989. "Asociación espacial entre individuos sanos y afectados por el secamiento del ciprés Austrocedrus chilensis", Bosque 10(2): 85-88.

ROTHKUGEL, M. 1916. Los bosques patagónicos. Ministerio de Agricultura de la Nación, Buenos Aires.

SALGUERO, J. 1990. Evaluación para el desarrollo de actividades primarias múltiples y sostenidas en la margen norte del río Manso Inferior. Tesis de grado. Universidad Nacional del Comahue, Centro Regional Universitario Bariloche, 98 pp.

SCHLEGEL, F. 1962. "Hallazgo de un bosque de cipreses cordilleranos en la provincia de Aconcagua", Boletín de la Universidad de Chile 32: 43-46.

SEIBERT, P. 1982. Carta de vegetación de la región de El Bolsón y su aplicación a la planificación del uso de la tierra. Fundación para la Educación, la Ciencia y la Cultura, Buenos Aires.

SERRA, V., GAJARDO, R. y CABELlO, A. 1986. Ciprés de la Cordillera: especie vulnerable. CONAF Chile, Santiago.

SINGER, R. 1971. "Forest mycology and forest communities in South America. Il. Mycorrhiza sociology and fungus succession in the Nothofagus dombeyi-Austrocedrus chilensis woods of Patagonia", US Department of Agriculture. Miscellaneous Publication 1189: 204-215.

THOMASSON, K. 1959. "Nahuel Huapi: Plankton of some lakes in an Argentina National Park, with notes on terrestrial vegetation", Acta Phytogeographica Suecica 42: 1-63.

TORTORELLI, L. 1956. Maderas y bosques argentinos. Editorial ACME. Buenos Aires.

VARSAVSKY, E., BETTUCCI, L., RODRIGUEZ, D. y GOMEZ, C. 1975. "Observaciones preliminares sobre la mortalidad del ciprés (Austrocedrus chilensis) en los bosques patagónicos", Publicación Fundación Bariloche 19. Bariloche.

VEBLEN, T. y LORENZ, D. 1987. "Post fire rodal development of Austrocedrus-Nothofagus forests in Northern Patagonia", Vegetatio 71: 113-126.

1988. "Recent vegetation changes along the forest/steppe ecotone of Northern Patagonia", Annales Association of American Geographers 78(1): 93-111. 